\title{
Plantar fasciitis and calcaneal spur formation are associated with abductor digiti minimi atrophy on MRI of the foot
}

\author{
Usha Chundru • Amy Liebeskind • Frank Seidelmann • \\ Joshua Fogel • Peter Franklin • Javier Beltran
}

Received: 10 December 2007 / Revised: 6 January 2008 / Accepted: 7 January 2008 /Published online: 20 February 2008

(C) ISS 2008

\begin{abstract}
Objective To determine the association of atrophy of the abductor digiti minimi muscle (ADMA), an MRI manifestation of chronic compression of the inferior calcaneal nerve suggesting the clinical diagnosis of Baxter's neuropathy, with MRI markers of potential etiologies, including calcaneal spur formation, plantar fasciitis, calcaneal edema, Achilles tendinosis and posterior tibial tendon dysfunction (PTTD).

Materials and methods Prevalence of calcaneal spur formation, plantar fasciitis, calcaneal edema, Achilles tendinosis and PTTD was assessed retrospectively on $100 \mathrm{MRI}$ studies with ADMA and 100 MRI studies without ADMA. Patients ranged in age from 10-92 years. Pearson chisquare analyses and Fisher's exact test were used to compare prevalence of the above findings in patients with and without ADMA. Logistic regression was used to determine which variables were significantly associated with ADMA.

Results Among patients with ADMA, there was significantly greater age $(57.2$ years vs 40.8 years, $\mathrm{p}<0.001)$,
\end{abstract}

U. Chundru $(\bowtie) \cdot$ A. Liebeskind $\cdot$ J. Fogel $\cdot$ J. Beltran Department of Radiology, Maimonides Medical Center, 4801 10th Avenue Maimonides Medical Center, Brooklyn, NY 11219, USA

e-mail: ushachundru@yahoo.com

A. Liebeskind $\cdot$ F. Seidelmann $\cdot$ P. Franklin $\cdot$ J. Beltran Franklin \& Seidelmann Subspecialty Radiology, Beachwood, Beachwood, OH, USA

J. Fogel

Department of Economics, Brooklyn College,

Brooklyn, NY, USA presence of Achilles tendinosis (22.0\% vs 3.0\%, $P<0.001)$, calcaneal edema $(15.0 \%$ vs $3.0 \%, P=0.005)$, calcaneal spur (48.0\% vs $7.0 \%, P<0.001)$, plantar fasciitis $(52.5 \%$ vs $11.0 \%, P<0.001)$, and PTTD (32.0\% vs $11.0 \%, P<0.001)$. After multivariate logistic regression analysis, only age [odds ratio (OR) 1.06, 95\% confidence interval (CI) 1.03, 1.09], calcaneal spur (OR 3.60, 95\% CI 1.28, 10.17), and plantar fasciitis (OR 3.35, 95\% CI 1.31, 8.56) remained significant.

Conclusion Advancing age, calcaneal spur, and plantar fasciitis are significantly associated with ADMA. Their high odds ratios support the notion of a possible etiologic role for calcaneal spur and plantar fasciitis in the progression to Baxter's neuropathy.

Keywords Foot · Muscle atrophy · Muscle denervation . Plantar fasciitis · MRI

\section{Introduction}

Entrapment of the first branch of the lateral plantar nerve (inferior calcaneal nerve), or Baxter's neuropathy, produces medial heel pain which may be indistinguishable from heel pain due to plantar fasciitis [1-3]. Baxter's neuropathy accounts for up to $20 \%$ of cases of heel pain [4] but is frequently overlooked as a potential cause of pain, even when pain persists after surgical intervention for other potential causes. On MRI, the presence of atrophy of the abductor digiti minimi muscle (ADMA) reflects chronic compression of the inferior calcaneal nerve and suggests the clinical diagnosis of Baxter's neuropathy $[1,5]$. Treatment for Baxter's neuropathy includes conservative therapy and, in cases in which non-interventional management is ineffective, surgical release [6]. 


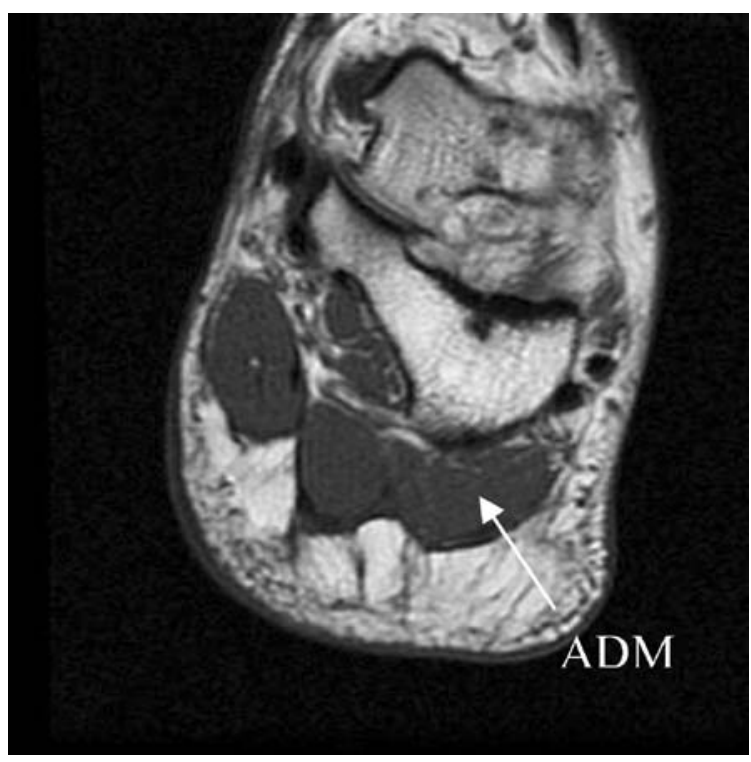

Fig. 1 Coronal T1-weighted image demonstrating normal abductor digiti minimi $(A D M)$ muscle

Entrapment of the inferior calcaneal nerve may result from altered biomechanics, reflected by posterior tibial tendon dysfunction (PTTD) or Achilles tendinosis, or may result from direct mechanical compression of the nerve due to plantar fasciitis and/or plantar calcaneal enthesophytes $[1,7]$. Our study was performed to assess the frequency of MRI manifestations of these potential etiologic factors in patients with and without ADMA to test the hypothesis that MRI findings reflecting altered biomechanics or direct compression of the inferior calcaneal nerve occur more frequently in patients with ADMA.

\section{Materials and methods}

\section{Patient population}

This retrospective study included 200 individuals who were referred for MRI of the hindfoot due to the presence of foot pain. These were individuals seen in a subspecialty teleradiology group practice with participant referrals from 40 states. The study was conducted with data from August 2006 to January 2007 and included patients of all ages. The study group was composed of 100 consecutive individuals with abductor digiti minimi atrophy and 100 consecutive individuals without abductor digiti minimi atrophy. No eligible participants were excluded. The subspecialty teleradiology group practice does not have an Institutional Review Board (IRB), and the study was conducted in compliance with the Declaration of Helsinki principles and the Health Insurance Portability and Accountability Act. Owing to the retrospective nature of the study, informed consent was not obtained.
Participants' ages ranged from 10-92 years [mean= 49.0 years, standard deviation $(\mathrm{SD})=16.9$ years] and included 139 women and 61 men.

\section{MRI technique}

Patients were scanned in magnets ranging from $0.2 \mathrm{~T}$ to 1.5 T. Long- and short-axis fat and fluid sensitive sequences were obtained without the administration of gadolinium. The technical parameters for all magnet strengths were as follows: sagittal T1-weighted, sagittal short-tau inversion recovery (STIR), oblique axial STIR, coronal T1-weighted, coronal STIR, plantar flexed axial proton density, axial T2weighted, and axial T2-weighted with fat saturation or STIR.

\section{Data acquisition}

In our retrospective analysis, potential patients were identified through review of reports of consecutive MRI studies obtained over a 6-month period. One hundred patients with ADMA and 100 patients without ADMA were selected for inclusion through review of reports, with subsequent confirmation of the presence or absence of ADMA through review of images. Patients were identified by review of MRI reports, with subsequent review of MR images. We reviewed 1,780 MRI reports in order to identify 100 patients with ADMA. Two radiologists (U.C., and A. L.), each with 4.5 years of experience of MRI interpretation and who were blinded to the initial interpretation, by consensus interpreted the MR images of both groups. Each MR image was evaluated for presence and absence of

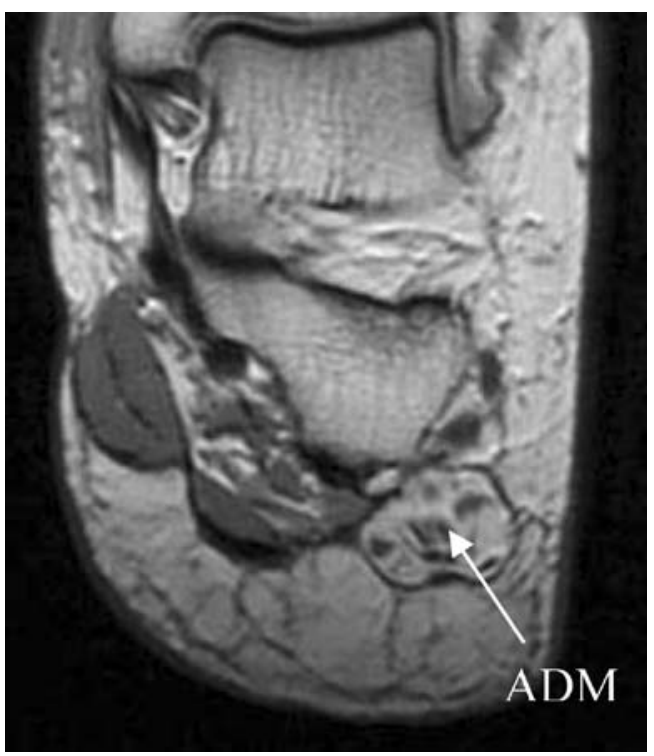

Fig. 2 Coronal T1-weighted image demonstrating fatty replacement of the abductor digiti minimi muscle $(A D M)$, indicating atrophy 
plantar fasciitis, calcaneal spur formation, calcaneal edema, PTTD, and Achilles tendon dysfunction. Atrophy was defined by the presence of any amount of fatty replacement of muscle mass on T1-weighted images (Figs. 1 and 2). Plantar fasciitis was defined by thickening, partial or full thickness tear of the plantar fascia. Calcaneal spur formation was defined by the presence of a bony outgrowth at the medial calcaneal tuberosity. Calcaneal edema was defined by increased signal intensity within the calcaneus adjacent to the insertion of the plantar fascia on fluid-sensitive sequences. PTTD was defined by tendon thickening, tenosynovitis or partial or full thickness tear. Achilles tendon dysfunction was defined by tendon thickening, peritendonitis or partial or full thickness tear.

\section{Statistical analysis}

Age and gender distribution, as well as the frequency of plantar fasciitis, calcaneal spur formation, calcaneal edema, PTTD, and Achilles tendon dysfunction, were compared in patients with and without ADMA. Age was compared with analysis of variance (ANOVA). For the categorical variables we used, as appropriate, Pearson chi-square analyses or Fisher's exact test to compare the proportion of those with atrophy to those without. We used logistic regression to determine odds ratios and 95\% confidence intervals for each of the variables to atrophy. First, we included each variable in a univariate model to determine its relationship to atrophy. We then simultaneously included all the variables in a multivariate analysis to determine the relationship of the variable to atrophy, while simultaneously adjusting for all the other variables. Alpha level was at 0.05 , and all tests were two-sided. SPSS version 15.0 (2006) (SPSS, Chicago, USA) was used for the analyses.

\section{Results}

Overall, of 200 patients in the study (100 with ADMA and 100 without ADMA), 25 (12.5\%) had Achilles tendinosis, $18(9 \%)$ had calcaneal edema $(n=18), 55(27.5 \%)$ had calcaneal spurs, 41 had other tendon abnormalities, 63 (31.5\%) had plantar fasciitis, and $43(21.5 \%)$ had PTTD. Fourteen $(7 \%)$ patients had isolated ADMA in the absence of any other identified pathological condition.

Comparison of the frequency of the above findings in patients with and without ADMA is shown in Table 1. Those with ADMA were significantly older than those without atrophy $(P<0.001)$. There were no gender differences.

Patients with atrophy compared to those without atrophy had significantly greater proportions of Achilles tendinosis, calcaneal edema, calcaneal spur, plantar fasciitis, and PTT dysfunction.
Table 1 Comparisons of demographic and clinical variables for those with and without atrophy of the abductor digiti minimi muscle

\begin{tabular}{|c|c|c|c|}
\hline Variable & $\begin{array}{l}\text { No atrophy }(n=100) \\
\% \text { (no.) }\end{array}$ & $\begin{array}{l}\text { Atrophy }(n=100) \\
\% \text { (no.) }\end{array}$ & $P$ \\
\hline Age (years) ${ }^{\mathrm{a}}$ & & & $<0.001$ \\
\hline [Mean (SD)] & $40.8(15.8)$ & $57.2(13.7)$ & \\
\hline Gender & & & 0.28 \\
\hline Women & $66.0 \%(66)$ & $73.0 \%(73)$ & \\
\hline Men & $34.0 \%(34)$ & $27.0 \%(27)$ & \\
\hline $\begin{array}{l}\text { Achilles } \\
\text { tendinosis }\end{array}$ & & & $<0.001$ \\
\hline No & $97.0 \%(97)$ & $78.0 \%(78)$ & \\
\hline Yes & $3.0 \%(3)$ & $22.0 \%(22)$ & \\
\hline Calcaneal edema & & & 0.005 \\
\hline No & $97.0 \%(97)$ & $85.0 \%(85)$ & \\
\hline Yes & $3.0 \%(3)$ & $15.0 \%(15)$ & \\
\hline Calcaneal spur & & & $<0.001$ \\
\hline No & $93.0 \%(93)$ & $52.0 \%(52)$ & \\
\hline Yes & $7.0 \%(7)$ & $48.0 \%(48)$ & \\
\hline $\begin{array}{l}\text { Other tendon } \\
\text { abnormality }\end{array}$ & & & 0.60 \\
\hline No & $81.0 \%(81)$ & $78.0 \%(78)$ & \\
\hline Yes & $19.0 \%(19)$ & $22.0 \%(22)$ & \\
\hline Plantar fasciitis ${ }^{\mathrm{b}}$ & & & $<0.001$ \\
\hline No & $89.0 \%(89)$ & $47.5 \%(47)$ & \\
\hline Yes & $11.0 \%(11)$ & $52.5 \%(52)$ & \\
\hline \multicolumn{4}{|l|}{ PTTD } \\
\hline No & $89.0 \%(89)$ & $68.0 \%(68)$ & \\
\hline Yes & $11.0 \%(11)$ & $32.0 \%(32)$ & $<0.001$ \\
\hline
\end{tabular}

${ }^{a}$ Mean and SDs compared with ANOVA, since continuous variable. All other analyses with Pearson's chi-square, except for Achilles tendinosis and calcaneal edema, which were with Fisher's exact test due to small subgroups

${ }^{\mathrm{b}}$ Data missing for one individual with atrophy

Table 2 shows logistic regression analyses for the variables. In univariate analyses, increased age, presence of Achilles tendinosis, calcaneal edema, calcaneal spur, plantar fasciitis, and PTT dysfunction were all significantly associated with increased odds ratios for atrophy. In the multivariate analysis, only increased age, presence of calcaneal spur, and presence of plantar fasciitis were significantly associated with atrophy. Presence of Achilles tendinosis only had a $P$ value of 0.09 , and calcaneal edema and PTT dysfunction had non-significant $P$ values. The multivariate analysis was also repeated, using data only from the individuals older than 21 years, in case inclusion of data from patients younger than 21 years $(n=15)$ had affected the results; the odds ratios, $P$ values and significance levels were almost exactly the same.

Of the 200 patients in the study, the consensus reviewers (U.C. and A.L) disagreed with findings in the initial report of eight, three with ADMA and five without ADMA. In two of the cases of ADMA, the presence of a calcaneal spur was not mentioned in the initial report. In the third case of 
Table 2 Logistic regression analyses for atrophy of the abductor digiti minimi muscle (OR odds ratio, $C I$ confidence interval)

\begin{tabular}{|c|c|c|c|c|}
\hline Variable & Univariate OR $(95 \% \mathrm{CI})$ & $P$ & Multivariate OR $(95 \% \mathrm{CI})$ & $P$ \\
\hline Age (years) & $1.08(1.05,1.11)$ & $<0.001$ & $1.06(1.03,1.09)$ & $<0.001$ \\
\hline Gender & & 0.28 & & 0.51 \\
\hline Women & 1.00 & & 1.00 & \\
\hline Men & $0.72(0.39,1.32)$ & & $0.75(0.33,1.73)$ & \\
\hline Achilles tendinosis & & $<0.001$ & & 0.09 \\
\hline No & 1.00 & & 1.00 & \\
\hline Yes & $9.12(2.63,31.60)$ & & $3.69(0.81,16.88)$ & \\
\hline Calcaneal edema & & 0.007 & & 0.47 \\
\hline No & 1.00 & & 1.00 & \\
\hline Yes & $5.71(1.60,20.39)$ & & $1.89(0.35,10.19)$ & \\
\hline Calcaneal spur & & $<0.001$ & & 0.02 \\
\hline No & 1.00 & & 1.00 & \\
\hline Yes & $12.26(5.18,29.10)$ & & $3.60(1.28,10.17)$ & \\
\hline Other tendon abnormality & & 0.60 & & 0.71 \\
\hline No & 1.00 & & 1.00 & \\
\hline Yes & $1.20(0.60,2.39)$ & & $1.19(0.48,2.97)$ & \\
\hline Plantar fasciitis & & $<0.001$ & & 0.01 \\
\hline No & 1.00 & & 1.00 & \\
\hline Yes & $8.95(4.27,18.77)$ & & $3.35(1.31,8.56)$ & \\
\hline PTTD & & 0.001 & & 0.31 \\
\hline No & 1.00 & & 1.00 & \\
\hline Yes & $3.81(1.79,8.10)$ & & $1.72(0.60,4.88)$ & \\
\hline
\end{tabular}

ADMA, the presence of posterior tibial tendinosis was not mentioned in the initial report. For three of the five patients without ADMA, the presence of a calcaneal spur was not mentioned in the initial report. For one of the patients without ADMA, the presence of Achilles tendinosis was not mentioned in the initial report. For one patient without ADMA, the presence of plantar fasciitis was not mentioned.

\section{Discussion}

Patients with ADMA had a significantly greater frequency of Achilles tendinosis, calcaneal edema, calcaneal spur, plantar fasciitis, and posterior tibialis tendon dysfunction than those without ADMA. After logistic regression analysis, only increased age, calcaneal spur, and plantar fasciitis remained significantly associated with ADMA.

The inferior calcaneal nerve supplies motor branches to the flexor digitorum brevis, quadratus plantae and abductor digiti minimi muscles and sensory fibers to the long plantar ligament and calcaneal periosteum [1]. The inferior calcaneal nerve originates from the lateral plantar nerve near the trifurcation of the posterior tibial nerve at the level of the medial malleolus and courses between the abductor hallucis and quadratus plantae muscles [8] (Figs. 3 and 4). When it reaches the inferior border of the abductor hallucis muscle, it turns laterally, passing anterior to the medial calcaneal tuberosity (or spur, if present), between the quadratus and underlying flexor digitorum brevis muscles until it reaches the abductor digiti minimi muscle [7, 9-11] (Fig. 3). The orthopedics and podiatrics literature suggests two possible sites of entrapment of the inferior calcaneal nerve which might result in Baxter's neuropathy: first, in patients with altered biomechanics, such as excessive pronation (flatfoot deformity), the nerve may be compressed as it turns laterally between the quadratus plantae and abductor hallucis muscles [12], and second, the nerve may be compressed as it passes anterior to the medial calcaneal tuberosity, or, if present, plantar calcaneal spur [7, 8, 13]. Our study evaluated potential markers of inferior calcaneal nerve compression due to either etiology. PTTD and Achilles tendinopathy were assessed as markers of altered biomechanics which might predispose the individual to inferior calcaneal nerve compression. Calcaneal spur formation and plantar fasciitis were assessed as potential causes of inferior calcaneal nerve compression as the nerve passes anterior to the medial calcaneal tuberosity. The persistence of an association between calcaneal spur formation and plantar fasciitis after logistic regression analysis supports the importance of these factors in the etiology of Baxter's neuropathy. The presence of 14 patients with isolated ADMA without other abnormalities suggests that Baxter's neuropathy may also occur in the absence of mechanical entrapment secondary to altered biomechanics.

Baxter's neuropathy can produce chronic heel pain. Clinically, the symptoms of Baxter's neuropathy may be indistinguishable from those of plantar fasciitis, and the two 


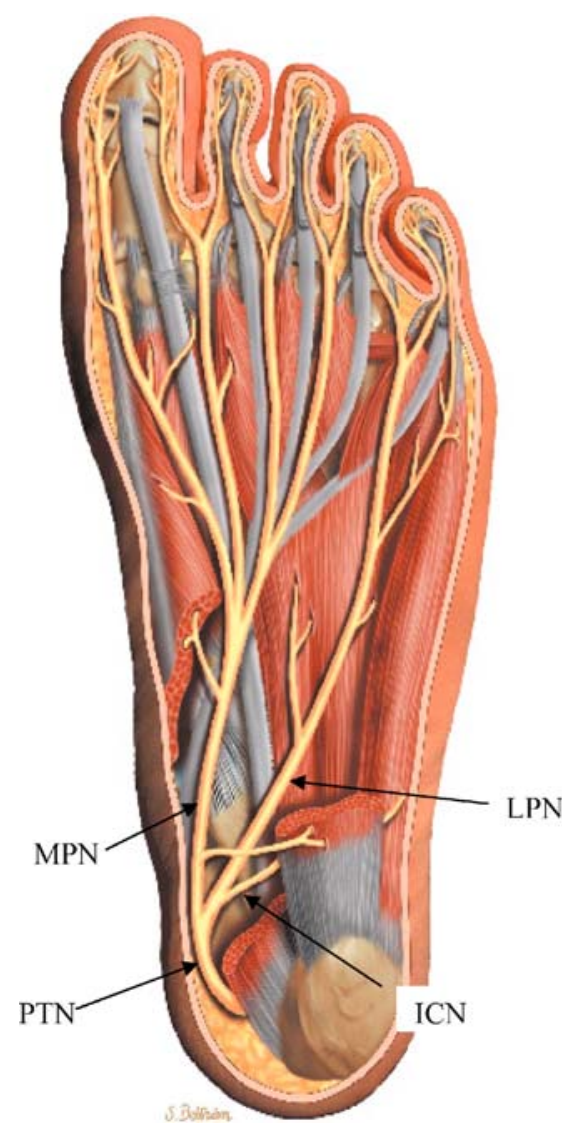

Fig. 3 An anatomic drawing of the sole of the foot after removal of the layers containing the skin, plantar fascia, and part of the flexor digitorum brevis muscle and abductor hallucis muscle cut away. Posterior tibial nerve trifurcation. $P T N$ posterior tibial nerve, $I C N$ inferior calcaneal nerve, $L P N$ lateral plantar nerve, $M P N$ medial plantar nerve

conditions are often superimposed $[5,7,14,15]$. Initial treatment for either condition is the same (taping to prevent abnormal motion, Achilles/plantar fascia stretching exercises, and medications such as steroids or non-steroidal anti-inflammatory agents [7]. Subsequent treatment includes injections and physical therapy, with surgical intervention reserved for patients who fail to respond to conservative measures.

For patients in whom heel pain persists despite conservative therapy, MRI evaluation is indicated prior to any surgical intervention. When a plantar fasciotomy is performed for clinically presumed plantar fasciitis in patients with occult Baxter's neuropathy, symptoms often persist, because at least a portion of the underlying cause of pain has not been addressed [14]. When pre-operative MRI demonstrates abductor digiti minimi atrophy, the surgeon is afforded the opportunity to advise the patient of the underlying abnormality and its prognosis, and, when indicated, to alter the surgical approach to include surgical release of the inferior calcaneal nerve.

The radiology literature rarely mentions ADMA as an MRI manifestation of Baxter's neuropathy. One study identified ten subjects with MRI evidence of ADMA out of 476 subjects who underwent MRI evaluation of the foot or ankle and found that ADMA was associated with obesity and the presence of a heel spur [5]. Another study evaluated patterns of muscle atrophy on MRI of the foot and found that, of 29 patients with focal denervation atrophy involving the muscles of the foot, 19 had ADMA. Of patients with denervation in the lateral plantar nerve distribution, 18 had atrophy involving the ADM and one had atrophy of the adductor hallucis muscle [16]. The largest study to date was a prospective evaluation of the prevalence of ADMA on MRI, which found ADMA in $6.3 \%$ of 602 consecutive patients who underwent MRI of the foot and ankle [17]. The prevalence of ADMA in that study is corroborated by the findings in our study, in which it was necessary for us to review 1,780 MRI studies in order to identify 100 patients with ADMA, corresponding to a prevalence of $5.6 \%$. The study also found that the most common associated findings in patients with ADMA were plantar enthesophytes and plantar fasciitis, but it did not include a statistical analysis of the prevalence of associated findings in patients with and without ADMA. Our study includes the largest number of patients with ADMA on MRI and is the first study to perform logistic regression analysis to determine independent variables associated with ADMA.

Limitations of the study include retrospective data collection, which may lead to selection bias; however, we attempted to minimize selection bias by including 100

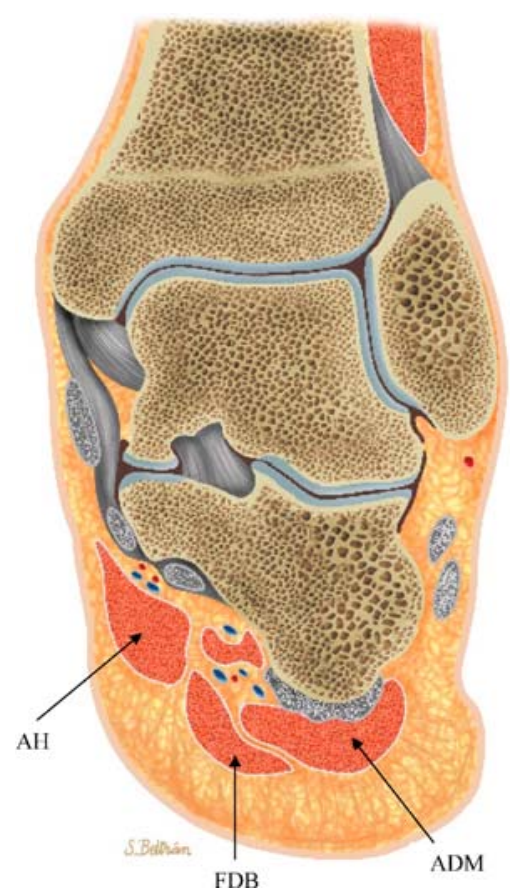

Fig. 4 An anatomic drawing of a coronal section through the right talocrural and talocalcaneal joints $(A D M$ abductor digiti minimi muscle, $F D B$ flexor digitorum brevis muscle, $A H$ abductor hallucis muscle) 
consecutive patients in each study group. In addition, the inclusion of 15 pediatric patients might have affected the results, as younger patients are less likely to have degenerative conditions of the foot or ankle and could potentially have falsely decreased the frequency of findings in the group of patients without abductor digiti minimi atrophy. However, a repeat statistical analysis, from which the pediatric patients had been excluded, demonstrated no significant difference in results.

In summary, our study showed a significant association between atrophy of the abductor digit minimi muscle, an MRI manifestation of Baxter's neuropathy, with age, plantar calcaneal spur formation, and plantar fasciitis. These findings support the notion of an etiologic role for compression of the inferior calcaneal nerve as it passes anterior to the medial calcaneal tuberosity in the development of Baxter's neuropathy.

\section{References}

1. Delfaut EM, Demondion X, Bieganski A, Thiron M-C, Mestdagh H, Cotton A. Imaging of foot and ankle nerve entrapment syndromes: from well-demonstrated to unfamiliar sites. Radiographics 2003; 23 : 613-623.

2. Oztuna V, Ozge A, Eskandari MM, Colak M, Golpinar A, Kuyurtar F. Nerve entrapment in painful heel syndrome. Foot Ankle Int 2002; 23: 208-211.

3. Przylucki H, Jones CL. Entrapment neuropathy of muscle branch of lateral plantar nerve: a cause of heel pain. J Am Podiatr Assoc 1981; 71: 119-124.

4. Baxter DE. Release of the nerve to the abductor digiti minimi. In: Kitaoka HB, ed. Master techniques in orthopaedic surgery of the foot and ankle. Philadelphia, PA: Lippincott Williams and Wilkins; 2002: 359.

5. Stanczak JD, McLean VA, Yao L. Atrophy of the abductor digiti minimi muscle: marker of neuropathic heel pain syndrome (abstract). Radiology 2001; 221(P): 522.

6. Baxter DE, Pfeffer GB. Treatment of chronic heal pain by surgical release of the first branch of the lateral plantar nerve. Clin Orthop 1992; 279: 229-236.

7. Offutt $\mathrm{S}$, DeHeer P. How to address Baxter's nerve entrapment. Podiatry Today 2004; 17: 52-58.

8. Louisia S, Masquelet AC. The medial and inferior calcaneal nerves: an anatomic study. Surg Radiol Anat 1999; 21: 169-173.

9. Arenson DJ, Cosentino GL, Suran SM. The inferior calcaneal nerve: an anatomical study. J Am Podiatr Assoc 1980; 70: 552.

10. Unlu RE, Orbay H, Kerem M, Firat Esmer A, Tuccar E, Sensoz O. Innervation of three weight-bearing areas of the foot: an anatomic study and clinical implications. J Plast Reconstr Aesthet Surg 2007 Mar 30 [Epub ahead of print\}.

11. Del Sol M, Olave E, Gabrielli C, Mandiola E, Prates JC. Innervation of the abductor digiti minimi muscle of the human foot: anatomical basis of the entrapment of the abductor digiti minimi nerve. Surg Radiol Anat 2002; 24: 18-22.

12. Rondhuis JJ, Huson A. The first branch of the lateral plantar nerve and heel pain. Acta Morphol Neerl Scand 1986; 24: 269-279.

13. Baxter DE, Thigpen CM. Heel pain-operative results. Foot Ankle 1984; 5: 16.

14. Barrett SL. A guide to neurogenic etiologies of heel pain. Podiatry Today 2005; 18: 36-44.

15. Fredericson M, Standage S, Chou L, Matheson G. Lateral plantar nerve entrapment in a competitive gymnast. Clin J Sport Med 2001; 11: 111-114.

16. Rajmane K, Schweitzer M, Morrison W. Medial and lateral plantar nerve denervation: MR patterns of motor atrophy. Presented at RSNA 2003, New York, NY.

17. Recht MP, Groof P, Ilaslan H, Recht HS, Sferra J, Donley BG. Selective atrophy of the abductor digiti quinti: an MRI study. AJR Am J Roentgenol 2007; 189: W123-W127. DOI 10.2214/ AJR.07.2229. 